\title{
Serial Radiographs Showing Progression of a Patellar Stress Fracture and Beneficial Surgical Technique for a Displaced Patellar Stress Fracture
}

\author{
Kazuha Kizaki, MD, Fumiharu Yamashita, MD, and Noboru Funakoshi, MD \\ Department of Orthopaedic Surgery and Rheumatology, Kyoto Shimogamo Hospital, Kyoto, Japan
}

\begin{abstract}
Stress fractures of the patella occurring in athletes are quite rare and only 23 cases have been introduced in English literatures. Stress fractures of the patella are classified into two types: transverse and longitudinal. They are mostly seen in the distal one-third of the patella on a plain lateral radiograph. We present here a 16-year-old female basketball player with a stress fracture of the patella. Her serial radiographs demonstrated progression of the fracture including a lytic cortex lesion, known as the gray cortex sign for early stage stress fractures, in the distal one-third of the patella on plain lateral radiographs. In addition, we introduce a surgical technique using non-absorbable suture ligatures (No.2 Ethibond) for a displaced transverse stress fracture of the patella when the extensor mechanism is intact. The ligatures are passed through into the patella using suture passers and simply tied down over the top of the patella. In our case, it led to neither hardware-related nor circumferential loop-related irritation postoperatively.
\end{abstract}

Keywords: Patella, Fracture, Stress

We often encounter stress fractures among athletes in primary care settings. In a study of 320 athletes, the tibia (49.1\%), tarsals (25.3\%) and metatarsals (8.8\%) were most predominantly affected by stress fractures ${ }^{1)}$. However, stress fractures of the patella are quite rare. Only 23 case reports of stress fractures of the patella in athletes have been introduced in the English literature since Devas $^{2)}$ first described in 1960 . They have been classified into two different types: transverse and longitudinal stress fractures of the patella ${ }^{3)}$. Transverse stress fractures of the patella were well described with 17 cases. For displaced transverse fractures, open reduction and internal fixation can be considered with tension

Received June 26, 2017; Revised August 13, 2017;

Accepted September 18, 2017

Correspondence to: Kazuha Kizaki, MD

Department of Orthopaedic Surgery and Rheumatology, Kyoto

Shimogamo Hospital, 17 Shimogamo, Sakyo-ku, Kyoto 606-0866, Japan

Tel: +81-75-781-1158, Fax: +81-75-722-2306

E-mail:kazuhakizaki@gmail.com

This is an Open Access article distributed under the terms of the Creative Commons Attribution Non-Commercial License (http://creativecommons.org/licenses/by-nc/4.0/) which permits unrestricted non-commercial use, distribution, and reproduction in any medium, provided the original work is properly cited. band wiring or screw fixation ${ }^{3)}$, though these techniques potentially cause postoperative hardware-related irritation.

\section{Case Report}

The patient was a 16-year-old female high school student who actively played basketball. Initially, she complained of right knee pain especially on the medial side when she visited her family doctor. On the first visit, radiographs were taken and the knee was considered intact as shown in Fig. 1A. On physical examination, there was no evidence of increasing synovial fluid. In spite of the right knee pain, she kept playing basketball. Three months later, her right knee pain aggravated and she took a week off from practice before resuming basketball. One more month later, when she played basketball in a competition, she felt her right knee cracking but continued playing in the game. Two days later, she visited the family doctor and from the radiographs, she was diagnosed with a displaced fracture of the patella with sclerosis at the margin of the fracture site as seen in Fig. 1A. At that time, she was able to stretch out her right knee.

Considering her high activity level and $>3 \mathrm{~mm}$ displacement of the distal fragment, open reduction and internal fixation were 

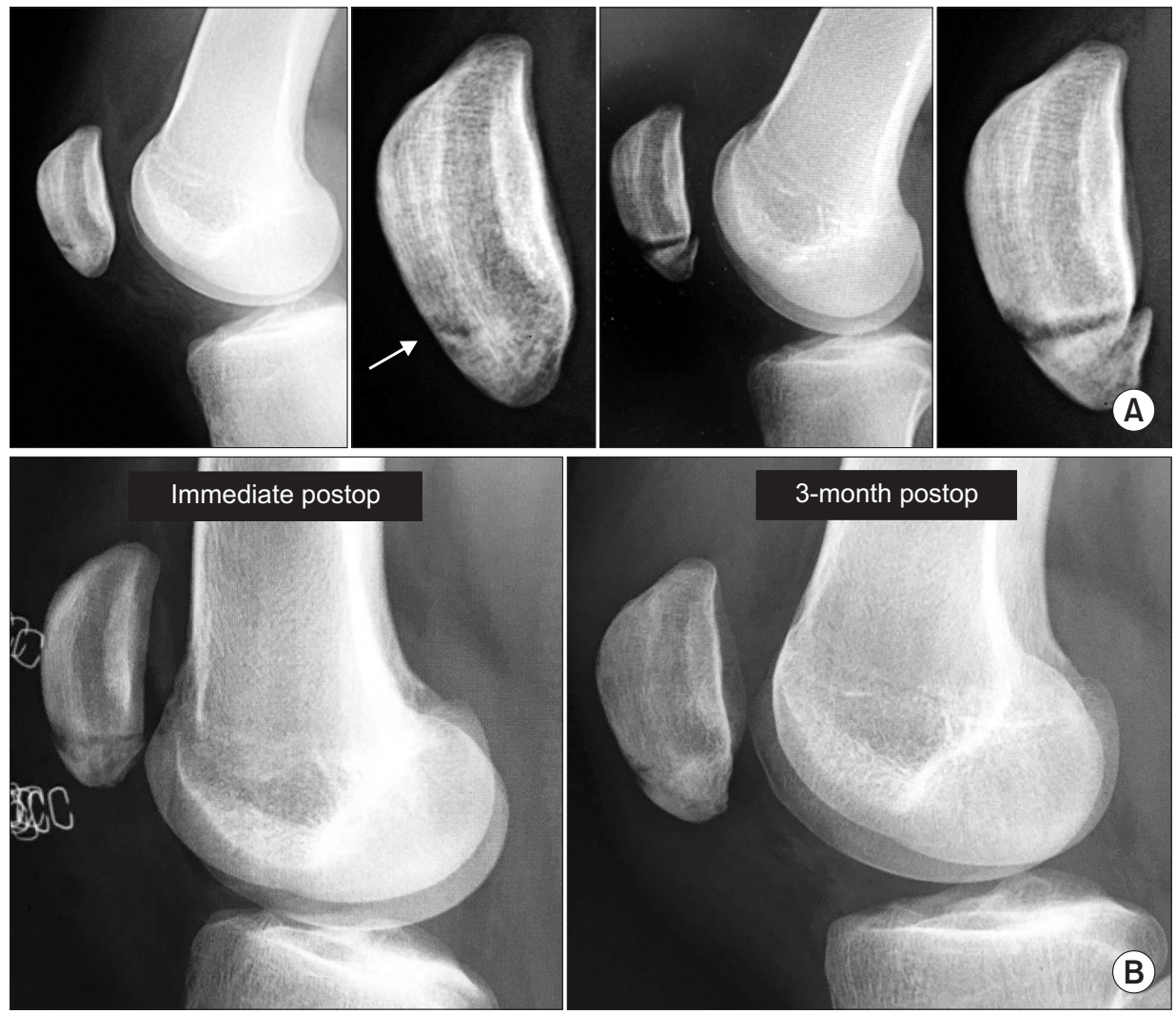

(B)
Fig. 1. Serial Radiographs showing the progression of a patellar stress fracture. (A) An undisplaced stress fracture of the patella is shown on the leftmost image. A radiolucent area (cortex lytic lesion known as "gray cortex sign") in the distal $1 / 3$ of the patella is seen (arrow), suggesting early stage of the stress fracture. The displaced stress fracture of the patella is also demonstrated on the remaining two images with the sclerotic fracture edges. (B) Postoperative (postop) $\mathrm{X}$-rays taken immediately postoperatively (left) and 3 months postoperatively (right).
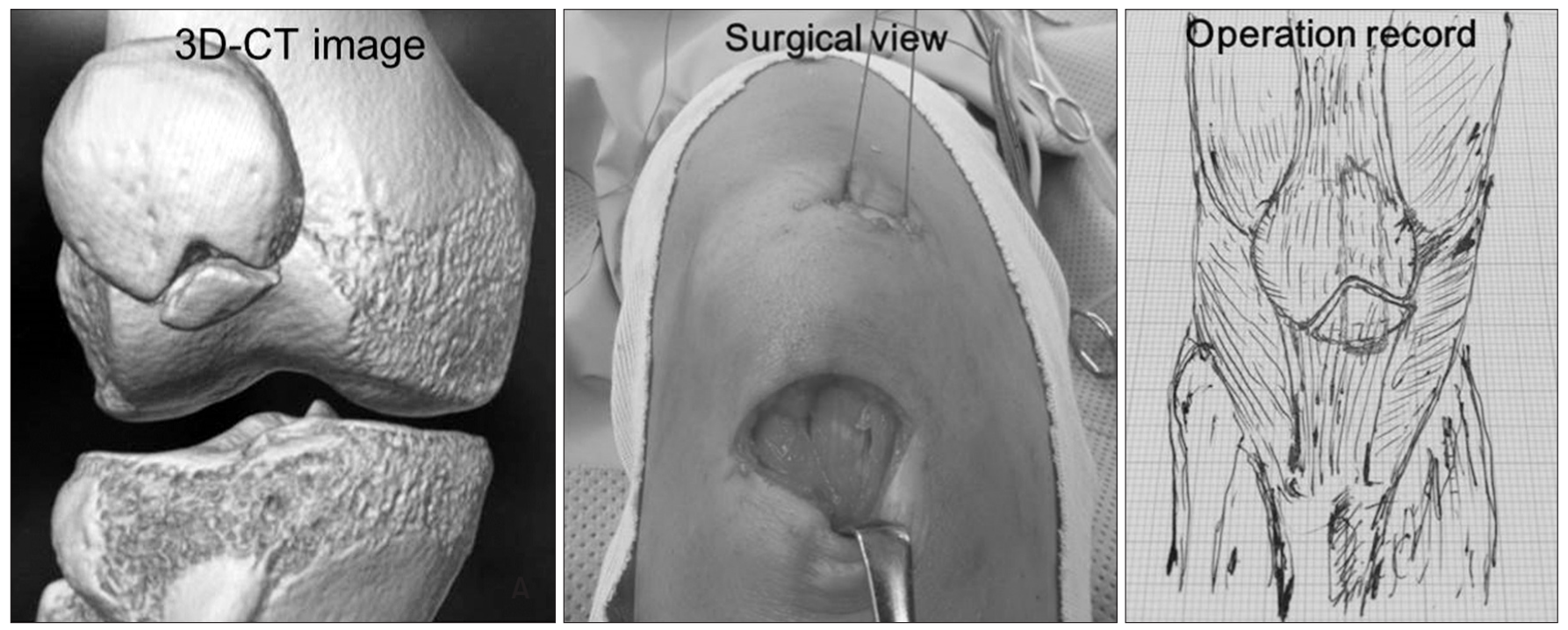

Fig. 2. Three-dimensional computed tomography (3D-CT) image, intraoperative photo and illustration showing the surgical technique of simple suturing for a displaced stress fracture of the patella. The 3D-CT image shows the fracture type is inverted V-shape. The surgical technique is demonstrated in the surgical view and the operation record: No.2 Ethibond (Ethicon) is passed though into the patella and simply tied down on top of the patella.

determined to be performed. An anterior transverse incision was made over the distal patella, and we found the extensor mechanism was not ruptured. By splitting the patella retinaculum longitudinally, the surface of the fracture was exposed. The sclerotic fracture edges were also directly observed intraoperatively. The sclerotic fracture margins were debrided and the bone fragment and the patella were longitudinally penetrated with a $2 \mathrm{~mm} \mathrm{~K}$ wire for making 2 holes. Non-absorbable suture ligatures, No.2 
Ethibond (Ethicon, Somerville, NJ, USA), were passed through with suture passers and tied down over the top of the patella as displayed in Fig. 2.

Postoperative care included full weight bearing with knee extension supported by an extension knee brace, knee flexion restricted to $45^{\circ}$ until three weeks and then to $90^{\circ}$ until eight weeks, after which full flexion was allowed. Three months postoperatively, the patient was allowed to return to training. Six months postoperatively she regained her preinjury activity level without any subsequent symptoms.

\section{Discussion}

Stress fractures often occur in athletes without adequate adaptation of the bone to the stress forces. By contrast, stress fractures of the patella are rare and not well discussed.

In this report, we described the general diagnostic and treatment process for possible patellar stress fractures ${ }^{4}$. For athletes with prolonged anterior knee pain in weight bearing and flexionextension motion, we do history taking to identify the duration of knee pain and physical examination to evaluate point tenderness, edema, warmth and ecchymosis on the superficial area of the patella. Then, if the symptom prolongs over a period of days to weeks and abnormality is found in physical exam, we obtain anterior-posterior, lateral and skyline radiographic views to evaluate the presence of resorption, sclerosis and callus in the patella.

If resorption is found without any true fracture line, we consider it as stress reaction, the initial stage of bone failure. If sclerosis is recognized, stress fracture is diagnosed. As a differential diagnosis, we have to be cautious about chronic symptomatic bipartite patellae and Sinding-Larsen-Johansson disease ${ }^{5)}$. In the absence of specific findings in radiography, we order computed tomography (CT) scanning and magnetic resonance imaging (MRI) to clarify evidence of bone reactive changes. In general, if patellar stress fracture dislocation is over $3 \mathrm{~mm}$, the articular incongruity is over $2 \mathrm{~mm}$ or extensor mechanism is impaired with loss of active knee extension, for which surgical treatment should be considered ${ }^{6}$. Currently, tension band wiring and screw fixation are the gold standard, but these techniques frequently cause hardware-related irritation postoperatively.

In our case, simple suture repair was selected with No.2 Ethibond passed though into the fragments and tied down to attach the fragments. It did not cause any irritation under the surface of the anterior patellar and inferior patellar poles. Although the non-absorbable suture technique using 5 Ti-Cron (Davis and Geck, Gosport, UK) has already been introduced in the fixation of patellar fractures, it left soft tissue irritation in 2 cases out of 7 cases $^{7}$. We assumed that soft tissue irritation in those cases might have occurred even with non-absorbable suturing over the anterior surface of patella or circumferential loop around the patella with a tension-band technique. In our case, non-absorbable suture ligatures were simply passed through into the fragments and tied down, which eventually lowered the contact areas between suture ligatures and soft tissues around the patella. Hence, we suggest that this simple suture technique for a displaced patellar stress fracture with the extensor mechanism intact is beneficial, especially for athletes who require smooth knee flexion movement without any irritation.

The mechanism of a patellar stress fracture is considered patellofemoral contact stress provoked from repetitive bending stress, which leads to increased tensile stress on the anterior surface of the patella with a subsequent microfracture and propagation ${ }^{3)}$ without extensor soft tissue rupture.

Sports activities such as basketball require repetitive knee flexion of $50^{\circ}-60^{\circ}$ and pivot shift movement, which maximizes patellofemoral joint reaction force and overstress on the middle to distal $1 / 3$ articular surface of the patella ${ }^{3}$. As results, previous reports revealed that almost all transverse stress fractures of the patella were seen on the middle to distal $1 / 3$ of the patella, predominantly on the distal $1 / 3$.

In stress fractures of the tibia, lytic lesions on anterior cortex were seen in the early stage of stress fractures ${ }^{8}$ and named as the gray cortex sign ${ }^{9)}$. In a stress fracture of the patella, Crowther et al. ${ }^{10)}$ showed the lytic lesion on the anterior cortex of the patella.

In reference to the gray cortex sign and the mechanism of stress fracture of the patella, we retrospectively reviewed plain radiographs taken on the first visit by a family physician and identified a radiolucent area on the distal $1 / 3$ of the patella, indicating disruption of the anterior cortical bone, as shown in Fig. 1. To date, for identification of early stage undisplaced stress fractures, MRI or radionuclide bone scan using technetium-99 m have been known to be very sensitive ${ }^{3}$, However, these imaging tests are not easy to access in primary care settings. We assume that a radiolucent area in the distal $1 / 3$ of the patella on a plain radiograph (lateral view), known as the gray cortex sign, helps screen stress fractures of the patella if the symptom prolongs over a period of days to weeks.

Although the fracture type in our case is quite similar to the transverse type, it may need to be classified as a new sub-type of the transverse stress fracture of the patella referring to the 3-dimensional CT (3D-CT) image as in Fig. 2. The shape of the fracture was inverted $\mathrm{V}$-shaped, though it was impossible to compare 
with previously reported cases because those were presented with only radiographs or 2-dimesinonal CT images.

In order to clearly categorize the fracture patterns of stress fractures of the patella, it is necessary for the fractures to be shown with 3D-CT images.

In summary, we demonstrated progression of a stress fracture of the patella with a lytic cortex lesion using serial radiographs, reflecting the early stage of a transverse stress fracture of the patella in the distal $1 / 3$ of the patella on a plain lateral radiograph. In addition, we proposed a simple but beneficial surgical technique using non-absorbable suture ligatures for displaced patellar fractures if the extensor mechanism is intact.

\section{Conflict of Interest}

No potential conflict of interest relevant to this article was reported.

\section{References}

1. Matheson GO, Clement DB, McKenzie DC, Taunton JE, Lloyd-Smith DR, MacIntyre JG. Stress fractures in athletes: a study of 320 cases. Am J Sports Med. 1987;15:46-58.

2. Devas MB. Stress fractures of the patella. J Bone Joint Surg Br. 1960;42:71-4.

3. Keeley A, Bloomfield P, Cairns P, Molnar R. Iliotibial band release as an adjunct to the surgical management of patellar stress fracture in the athlete: a case report and review of the literature. Sports Med Arthrosc Rehabil Ther Technol. 2009; $1: 15$.

4. Mayer SW, Joyner PW, Almekinders LC, Parekh SG. Stress fractures of the foot and ankle in athletes. Sports Health. 2014;6:481-91.

5. Kahanov L, Eberman LE, Games KE, Wasik M. Diagnosis, treatment, and rehabilitation of stress fractures in the lower extremity in runners. Open Access J Sports Med. 2015;6:8795.

6. Melvin JS, Karunakar MA. Patella fractures and extensor mechanism injuries. In: Court-Brown CB, Heckman JD, McQueen MM, Ricci WM, Tornetta P III, eds. Rockwood and Green's fractures in adults. Philadelphia: Wolters Kluwer; 2004. p2269-302.

7. Chatakondu SC, Abhaykumar S, Elliott DS. The use of nonabsorbable suture in the fixation of patellar fractures: a preliminary report. Injury. 1998;29:23-7.

8. Gaeta M, Minutoli F, Scribano E, Ascenti G, Vinci S, Bruschetta D, Magaudda L, Blandino A. CT and MR imaging findings in athletes with early tibial stress injuries: comparison with bone scintigraphy findings and emphasis on cortical abnormalities. Radiology. 2005;235:553-61.

9. Mulligan ME. The "gray cortex": an early sign of stress fracture. Skeletal Radiol. 1995;24:201-3.

10. Crowther MA, Mandal A, Sarangi PP. Propagation of stress fracture of the patella. Br J Sports Med. 2005;39:e6. 